Communications in Physics, Vol. 26, No. 1 (2016), pp. 1-9

DOI: $10.15625 / 0868-3166 / 26 / 1 / 7860$

\title{
ON THE CP VIOLATION PHASE IN A NEUTRINO MIXING MODEL WITH AN A4 FLAVOR SYMMETRY
}

\author{
PHI QUANG VAN AND NGUYEN THI HONG VAN ${ }^{\dagger}$ \\ Institute of Physics, Vietnam Academy of Science and Technology \\ ${ }^{\dagger}$ E-mail: nhvan@iop.vast.vn \\ Received 09 March 2016 \\ Accepted for publication 14 April 2016
}

\begin{abstract}
Neutrino masses and mixing in an extended standard model acquiring an A4 flavor symmetry are considered. The corresponding three-neutrino mixing matrix obtained via a perturbative method allows us to determine the Dirac $C P$ violation phase $\left(\delta_{C P}\right)$ as a function of the mixing angles $\left(\theta_{12}, \theta_{23}, \theta_{13}\right)$. Then, numerical values and distributions of $\delta_{C P}$ are given. The latter values are quite close to the global fits of the experimental data for both the normal ordering and inverse ordering of the neutrino masses.
\end{abstract}

Keywords: neutrino mass and mixing, flavor symmetries, models beyond the standard model.

Classification numbers: 14.60.Pq, 11.30.Fs, 12.60.-i.

\section{INTRODUCTION}

Standard model (SM) [1-4] has been confirmed as an excellent model of elementary particles and their interactions, especially after the discovery of the Brout-Englert-Higgs boson (Higg boson) by ATLAS and CMS [5,6], which are the two biggest LHC collaborations (see [7] for a review on the discovering of the Higgs boson). The SM, however, cannot explain a number of phenomena including that of the neutrino masses and mixing $[8,9]$. They, therefore, call for an extension of the SM. One of the extensions of the SM which has attracted great interest for last ten years is that with a flavor symmetry. A widely investigated flavor symmetry is based on the discrete group A4 (see, for example, $[10,11])$. This symmetry can make a neutrino mixing scheme tribi-maximal (TBM). The recent experimental data [12,13], however, shows that the mixing angle $\theta_{13}$ and the Dirac CP-violation phase (CPV) $\delta_{C P}$ are non-zero. That means that the TBM scheme is no longer valid. Consequently, many attempts to explain these new phenomena have been made. Here, in this paper, as reported in [14], we use a perturbation method to diagonalize a general neutrino mass matrix and obtain a Pontecorvo-Maki-Nakagawa-Sakata (PMNS) neutrino mixing matrix from which a relation between $\delta_{C P}$ and the mixing angles $\theta_{i j}$ can be derived. This relation 
allows us, using experimental data of the mixing angles, to calculate $\delta_{C P}$ numerically, for both the normal ordering (NO) and inverse ordering (IO) of neutrino masses. This means that the present paper is an extended work of [14] in which only $\delta_{C P}$ for an NO was considered.

The plan of this paper is the following. In the next section we will make a quick introduction to the representations of A4 group and an A4-symmetric neutrino mass model. Section 3 deals with a perturbation approach to the problem of neutrino masses and mixing for this model, from where a mass spectrum and a relation between $\delta_{C P}$ and $\theta_{i j}$ can be obtained. Then, numerical values and distributions of $\delta_{C P}$ in both NO and IO are presented.

\section{NEUTRINO MASS MODEL WITH AN A4 FLAVOR SYMMETRY}

\section{Quick introduction to representations of A4 group}

Let us give a concise introduction to representations of A4 which are widely given in the literature (see, for example, $[15,16])$. This group is composed of 12 elements and generated by two basic permutations $\mathrm{S}$ and $\mathrm{T}$,

$$
S^{2}=T^{3}=(S T)^{3}=1 .
$$

It has a three-dimensional unitary representation generated by

$$
S=\left(\begin{array}{ccc}
1 & 0 & 0 \\
0 & -1 & 0 \\
0 & 0 & -1
\end{array}\right), \quad T=\left(\begin{array}{lll}
0 & 1 & 0 \\
0 & 0 & 1 \\
1 & 0 & 0
\end{array}\right)
$$

and three one-dimensional unitary representations $1,1^{\prime}$ and $1^{\prime \prime}$ generated by

$$
\begin{gathered}
1: S=1 \quad T=1, \\
1^{\prime}: S=1 \quad T=e^{i 2 \pi / 3} \equiv \omega, \\
1^{\prime \prime}: S=1 \quad T=e^{i 4 \pi / 3} \equiv \omega^{2} .
\end{gathered}
$$

As in the case of an arbitrary group, applications of A4 often require to know the multiplication rule of its (irreducible) representations

$$
\begin{gathered}
1 \times 1=1, \quad 1^{\prime} \times 1^{\prime \prime}=1, \quad 1^{\prime \prime} \times 1^{\prime}=1, \quad 1^{\prime} \times 1^{\prime}=1^{\prime \prime}, \quad 1^{\prime \prime} \times 1^{\prime \prime}=1^{\prime}, \\
3 \times 3=1+1^{\prime}+1^{\prime \prime}+3_{1}+3_{2} .
\end{gathered}
$$

Let us explain the rule (4b) in more details, while the first ones are trivial. For two triplets, say $3_{a} \sim\left(a_{1}, a_{2}, a_{3}\right)$ and $3_{b} \sim\left(b_{1}, b_{2}, b_{3}\right)$, their direct product can be decomposed into irreducible representations in the following way:

$$
\begin{gathered}
1=a_{1} b_{1}+a_{2} b_{2}+a_{3} b_{3}, \\
1^{\prime}=a_{1} b_{1}+\omega^{2} a_{2} b_{2}+\omega a_{3} b_{3}, \\
1^{\prime \prime}=a_{1} b_{1}+\omega a_{2} b_{2}+\omega^{2} a_{3} b_{3}, \\
3_{1} \sim\left(a_{2} b_{3}, a_{3} b_{1}, a_{1} b_{2}\right), \\
3_{2} \sim\left(a_{3} b_{2}, a_{1} b_{3}, a_{2} b_{1}\right) .
\end{gathered}
$$

The information given here is used in constructing an A4-invariant action (Lagrangian) of the considered model, including the Yukawa terms (6). 


\section{The model generality}

In principle, an A4 flavor symmetry can be applied to a neutrino mixing model with an arbitrary number of Majorana neutrinos but here we will consider, as an example, a model with three active Majorana neutrinos, for which the neutrino mass matrix is symmetric and parametrized by 6 independent parameters. Similar models have been studied widely before (see, for example, in [17-19]) but the main goal of this paper (and [14]) is to concentrate on considering the Dirac CPV phase (see also [20]). Besides the SM leptons transforming now also under A4 as a triplet (left-handed leptons $l_{L}, l=e, \mu, \tau$ ) or singlets (right-handed leptons $e_{R}, \mu_{R}$ and $\tau_{R}$ ), the lepton sector of this model includes an A4 triplet $N$ which is an $S U(2)_{L}$ singlet (right-handed neutrino). The scalar sector consists of the SM Higgs $\phi_{h}$ which is an A4 singlet, and five new $S U(2)_{L}$-singlet scalars including two A4-triplets (denoted as $\phi_{E}, \phi_{N}$ ) and three A4-singlets (denoted as $\xi, \xi^{\prime}, \xi^{\prime \prime}$ ). The A4- and $S U(2)_{L}$ transformation rules of the leptons and the scalars in the present model are listed in the table given below. It is enough for us to construct an A4-invariant Lagrangian.

Table 1. A4 and $S U(2)_{L}$ symmetry of leptons and scalars.

\begin{tabular}{|l||c|c|c|c|c|c|c|c|c|c|c|}
\hline & $l_{L}$ & $e_{R}$ & $\mu_{R}$ & $\tau_{R}$ & $N$ & $\phi_{E}$ & $\phi_{N}$ & $\xi$ & $\xi^{\prime}$ & $\xi^{\prime \prime}$ & $\phi_{h}$ \\
\hline $\mathrm{A} 4$ & 3 & 1 & $1^{\prime}$ & $1^{\prime \prime}$ & 3 & 3 & 3 & 1 & $1^{\prime}$ & $1^{\prime \prime}$ & 1 \\
\hline$S U(2)_{L}$ & 2 & 1 & 1 & 1 & 1 & 1 & 1 & 1 & 1 & 1 & 2 \\
\hline
\end{tabular}

The Lagrangian of the model includes the new Yukawa term

$$
\begin{aligned}
-\mathscr{L}_{Y}^{\text {new }}= & \lambda_{e}\left(\bar{l}_{L} \phi_{h}\right) e_{R} \frac{\phi_{E}}{\Lambda}+\lambda_{\mu}\left(\bar{l}_{L} \phi_{h}\right)^{\prime \prime} \mu_{R} \frac{\phi_{E}}{\Lambda}+\lambda_{\tau}\left(\bar{l}_{L} \phi_{h}\right)^{\prime} \tau_{R} \frac{\phi_{E}}{\Lambda}+\lambda_{N} \bar{l}_{L} \tilde{\phi}_{h} N \\
& +c_{N}\left(\overline{N^{c}} N\right) \phi_{N}+c_{\xi}\left(\overline{N^{c}} N\right) \xi+c_{\xi^{\prime}}\left(\overline{N^{c}} N\right)^{\prime \prime} \xi^{\prime}+c_{\xi^{\prime \prime}}\left(\overline{N^{c}} N\right)^{\prime} \xi^{\prime \prime}+\text { H.c. }
\end{aligned}
$$

From here, by denoting the VEVs of the scalars as

$$
\langle\xi\rangle=u_{a},\left\langle\xi^{\prime}\right\rangle=u_{b},\left\langle\xi^{\prime \prime}\right\rangle=u_{c},\left\langle\phi_{E}\right\rangle=\left(u_{1}, u_{2}, u_{3}\right),\left\langle\phi_{N}\right\rangle=\left(v_{1}, v_{2}, v_{3}\right),\left\langle\phi_{h}\right\rangle=v,
$$

the mass matrix of the charged leptons has the form

$$
M_{\text {lept }}=v\left(\begin{array}{ccc}
\kappa_{e} u_{1} & \kappa_{e} u_{2} & \kappa_{e} u_{3} \\
\kappa_{\mu} u_{1} & \omega \kappa_{\mu} u_{2} & \omega^{2} \kappa_{\mu} u_{3} \\
\kappa_{\tau} u_{1} & \omega^{2} \kappa_{\tau} u_{2} & \omega \kappa_{\tau} u_{3}
\end{array}\right)
$$

where

$$
\kappa_{e}=\frac{\lambda_{e}}{\Lambda}, \kappa_{\mu}=\frac{\lambda_{\mu}}{\Lambda}, \kappa_{\tau}=\frac{\lambda_{\tau}}{\Lambda} .
$$

To keep maximally the SM Higgs VEV structure, we can assume $\left\langle\phi_{E}\right\rangle=(u, u, u)$, thus, the charged lepton mass matrix becomes

$$
M_{\text {lept }}=U_{L}\left(\begin{array}{ccc}
y_{e} v & 0 & 0 \\
0 & y_{\mu} v & 0 \\
0 & 0 & y_{\tau} v
\end{array}\right) \equiv U_{L}\left(\begin{array}{ccc}
m_{e} & 0 & 0 \\
0 & m_{\mu} & 0 \\
0 & 0 & m_{\tau}
\end{array}\right),
$$


where

$$
\begin{gathered}
U_{L}=\frac{1}{\sqrt{3}}\left(\begin{array}{ccc}
1 & 1 & 1 \\
1 & \omega & \omega^{2} \\
1 & \omega^{2} & \omega
\end{array}\right), \\
y_{e}=u \kappa_{e}, y_{\mu}=u \kappa_{\mu}, y_{\tau}=u \kappa_{\tau},
\end{gathered}
$$

and $m_{e}, m_{\mu}, m_{\tau}$ are the charged lepton masses.

In the neutrino subsector, we obtain the following Majorana mass matrix

$$
M_{N}=\left(\begin{array}{ccc}
c_{a}+c_{b}+c_{d} & \varepsilon_{3} & \varepsilon_{2} \\
\varepsilon_{3} & c_{a}+\omega c_{b}+\omega^{2} c_{d} & \varepsilon_{1} \\
\varepsilon_{2} & \varepsilon_{1} & c_{a}+\omega^{2} c_{b}+\omega c_{d}
\end{array}\right) \equiv\left(\begin{array}{ccc}
a & b & c \\
b & e & d \\
c & d & f
\end{array}\right),
$$

where $c_{a}=c_{\xi} u_{a}, c_{b}=c_{\xi^{\prime}} u_{b}, c_{d}=c_{\xi^{\prime \prime}} u_{c}, \varepsilon_{1}=c_{N} v_{1}, \varepsilon_{2}=c_{N} v_{2}, \varepsilon_{3}=c_{N} v_{3}$, and Dirac mass matrix

$$
M_{D}=\left(\begin{array}{ccc}
\lambda_{N_{1}} v & 0 & 0 \\
0 & \lambda_{N_{2}} v & 0 \\
0 & 0 & \lambda_{N_{3}} v
\end{array}\right) \equiv\left(\begin{array}{ccc}
x & 0 & 0 \\
0 & y & 0 \\
0 & 0 & z
\end{array}\right)
$$

The seesaw mechanism gives us the neutrino mass matrix,

$$
M_{v}=-M_{D}^{T} M_{N}^{-1} M^{D}
$$

which, with (13) and (14) taken into account, can be written as

$$
M_{v}=\frac{-1}{\operatorname{det}(M)}\left(\begin{array}{ccc}
\left(d^{2}-e f\right) x^{2} & (-c d+b f) x y & (-b d+c e) x z \\
(-c d+b f) x y & \left(c^{2}-a f\right) y^{2} & (-b c+a d) y z \\
(-b d+c e) x z & (-b c+a d) y z & \left(b^{2}-a e\right) z^{2}
\end{array}\right),
$$

with $\operatorname{det}(M)=2 b c d-c^{2} e-b^{2} f-a\left(d^{2}-e f\right)$ being the determinant of $M_{N}$.

Diagonalizing a neutrino mass matrix, such as the one in (16), is often a difficult task. To solve this problem different methods and tricks have been proposed. Here we will use a perturbation approach to solving this problem.

\section{NEUTRINO MIXING AND THE CP VIOLATION PHASE}

Let us work in the basis where the lepton mass matrix is diagonal. In this basis $M_{v}$ becomes

$$
\mathbb{M}_{v}=U_{L}^{T} M_{v} U_{L} \equiv\left(\begin{array}{ccc}
A & B & C \\
B & E & D \\
C & D & F
\end{array}\right)
$$

The current experimental data have shown that the PMNS neutrino mixing matrix $U_{P M N S}$ is a small deviation from the TBM form and we have (see [14] and also [21])

$$
\mathbb{M}_{V}=M_{0}+\lambda V
$$

with

$$
M_{0}=\left(\begin{array}{ccc}
A & B & -B \\
B & E & -(A-E+B) \\
-B & -(A-E+B) & E
\end{array}\right), \quad \lambda V=\left(\begin{array}{ccc}
0 & 0 & e_{1} \\
0 & 0 & e_{3} \\
e_{1} & e_{3} & e_{2}
\end{array}\right)
$$


where $\lambda$ is a perturbation, i.e., small, parameter and $M_{0}$ is a non-perturbative (TBM level) mass matrix which can be diagonalized by the TBM neutrino mixing matrix

as

$$
U_{T B M}=\left(\begin{array}{ccc}
\sqrt{\frac{2}{3}} & \sqrt{\frac{1}{3}} & 0 \\
-\sqrt{\frac{1}{6}} & \sqrt{\frac{1}{3}} & \sqrt{\frac{1}{2}} \\
\sqrt{\frac{1}{6}} & -\sqrt{\frac{1}{3}} & \sqrt{\frac{1}{2}}
\end{array}\right) \times P \sim\left(\left|1^{0}\right\rangle,\left|2^{0}\right\rangle,\left|3^{0}\right\rangle\right) \times P
$$

$$
\operatorname{diag}\left(m_{1}^{0}, m_{2}^{0}, m_{3}^{0}\right)=U_{T B M}^{\dagger} M_{0} U_{T B M}
$$

Here $P$ is a matrix of Majorana phases which are not given explicitly because they play no role in the $\mathrm{CP}$ violation process. Using the perturbation expansion of the basis $|n\rangle$ around the nonperturbative one, $\left|n^{0}\right\rangle$,

$$
|n\rangle=\left|n^{0}\right\rangle+\lambda \sum_{k \neq n}\left|k^{0}\right\rangle \frac{V_{k n}}{m_{n}^{0}-m_{k}^{0}}+O\left(\lambda^{2}\right) ; \quad n, k=1,2,3
$$

where

$$
V_{k n}=\langle k|V| n\rangle,
$$

we can diagonalize the matrix $M_{v}$ by the PMNS matrix

$$
U_{P M N S}=\left(\begin{array}{ccc}
\sqrt{\frac{2}{3}}+\sqrt{\frac{1}{3}} X & \sqrt{\frac{1}{3}}-\sqrt{\frac{2}{3}} X & -\sqrt{\frac{2}{3}} Y-\sqrt{\frac{1}{3}} Z \\
-\sqrt{\frac{1}{6}}+\sqrt{\frac{1}{3}} X+\sqrt{\frac{1}{2}} Y & \sqrt{\frac{1}{3}}+\sqrt{\frac{1}{6}} X+\sqrt{\frac{1}{2}} Z & \sqrt{\frac{1}{2}}+\sqrt{\frac{1}{6}} Y-\sqrt{\frac{1}{3}} Z \\
\sqrt{\frac{1}{6}}-\sqrt{\frac{1}{3}} X+\sqrt{\frac{1}{2}} Y & -\sqrt{\frac{1}{3}}-\sqrt{\frac{1}{6}} X+\sqrt{\frac{1}{2}} Z & \sqrt{\frac{1}{2}}-\sqrt{\frac{1}{6}} Y+\sqrt{\frac{1}{3}} Z
\end{array}\right) \times P+\Delta U,
$$

where $\Delta U$ is a higher order perturbative correction to $U_{P M N S}$ and

$$
X=\frac{\sqrt{2}}{6}\left(\frac{2 e_{3}-e_{1}-e_{2}}{m_{1}^{0}-m_{2}^{0}}\right), Y=\frac{\sqrt{3}}{6}\left(\frac{2 e_{1}+e_{2}}{m_{1}^{0}-m_{3}^{0}}\right), Z=\frac{1}{\sqrt{6}}\left(\frac{e_{1}-e_{2}}{m_{2}^{0}-m_{3}^{0}}\right) .
$$

Here we discuss only the CP-violation Dirac phase $\delta_{C P}$, while the mass spectrum derived by the above-described diagonalization of the neutrino mass matrix $M_{v}$ is a subject of a future work because more analysis on the VEV's of the scalars and the Yukawa coupling coefficients as well as different phenomena and experimental results is needed. Since the CP violation is expected to be small the symmetric matrix $\mathbb{M}_{V}$ is near to a Hermitian matrix, therefore, the matrix (24) is near to an unitary one.

Denoting the matrix elements of (24) by $U_{i j}, i, j=1,2,3$, we get the relation

$$
U_{21}+\sqrt{2} U_{22}-U_{31}-\sqrt{2} U_{32}=2 U_{11}-\sqrt{2} U_{12}+\Delta U_{i j},
$$

where $\Delta U_{i j}$ is a higher order perturbative correction. Fitting the matrix (24) with the elements of the matrix $U_{P M N S}$ in the canonical form

$$
U_{P M N S}=\left(\begin{array}{ccc}
c_{12} c_{13} & s_{12} c_{13} & s_{13} e^{-i \delta} \\
-c_{23} s_{12}-s_{13} s_{23} c_{12} e^{i \delta} & c_{23} c_{12}-s_{13} s_{23} s_{12} e^{i \delta} & s_{23} c_{13} \\
s_{23} s_{12}-s_{13} c_{23} c_{12} e^{i \delta} & -s_{23} c_{12}-s_{13} c_{23} s_{12} e^{i \delta} & c_{23} c_{13}
\end{array}\right) \times P
$$


where $s_{i j}=\sin \theta_{i j}, c_{i j}=\cos \theta_{i j} ; i, j=1,2,3$, we obtain from (26) the following relation between $\delta_{C P} \equiv \delta$ and the mixing angles $\theta_{i j}$ :

$$
\cos \delta \tan \theta_{13}=\frac{\left(\sqrt{2}-\tan \theta_{12}\right)}{\left(1+\sqrt{2} \tan \theta_{12}\right)\left(1-\tan \theta_{23}\right)}\left(\frac{\sqrt{2}}{c_{23}}-\frac{1+\tan \theta_{23}}{c_{13}}\right) .
$$

Solving (28) for $\delta \in[0,2 \pi]$ we can get two solutions: if a value $\delta_{0}$ is a solution of (28), the value $2 \pi-\delta_{0}$ is the other solution. Therefore, we can choose to discuss one of these solutions, for example, the bigger one.

In general, both theoretically and experimentally, it is not very easy to find $\delta_{C P}$ but the relation (28) allows us to determine $\delta_{C P}$ numerically via the experimental data on the mixing angles $\theta_{i j}$. The distributions of $\delta_{C P}$ are plotted in Fig. 1 and Fig. 2 for a normal- and an inverseordering. Here, a value of $\delta_{C P}$ is calculated event by event using equation (28) with the value of $\sin \theta_{i j}$ taken randomly (within $3 \sigma$ range) based on a Gaussian distribution having the mean and the standard deviation $(\sigma)$ to be the best fit value and the sigmas, respectively, of $\sin \theta_{i j}$ determined experimentally and given in $[12,13]$. These figures show that $\delta_{C P}$ distributes in the region $3.1<\delta_{C P}<5.8$ (for an $\mathrm{NO}$ ) and $3.1<\delta_{C P}<6.3$ (for an IO). This distributions have a mean value at $\delta_{C P}=4.28$ (for an NO) and at $\delta_{C P}=4.56$ (for an IO) which is close, between $1 \sigma$, to the global fit values (GFV's) to the experimental data and have a maximum density around $\delta_{C P}=4.45$ (for an NO) and $\delta_{C P}=4.55$ (for an IO) between $1 \sigma$ region from the GFV $[12,13]$. For more information, $\delta_{C P}$ versus $\sin ^{2} \theta_{13}$ are depicted in Fig. 3 for an NO and in Fig. 4 for an IO.

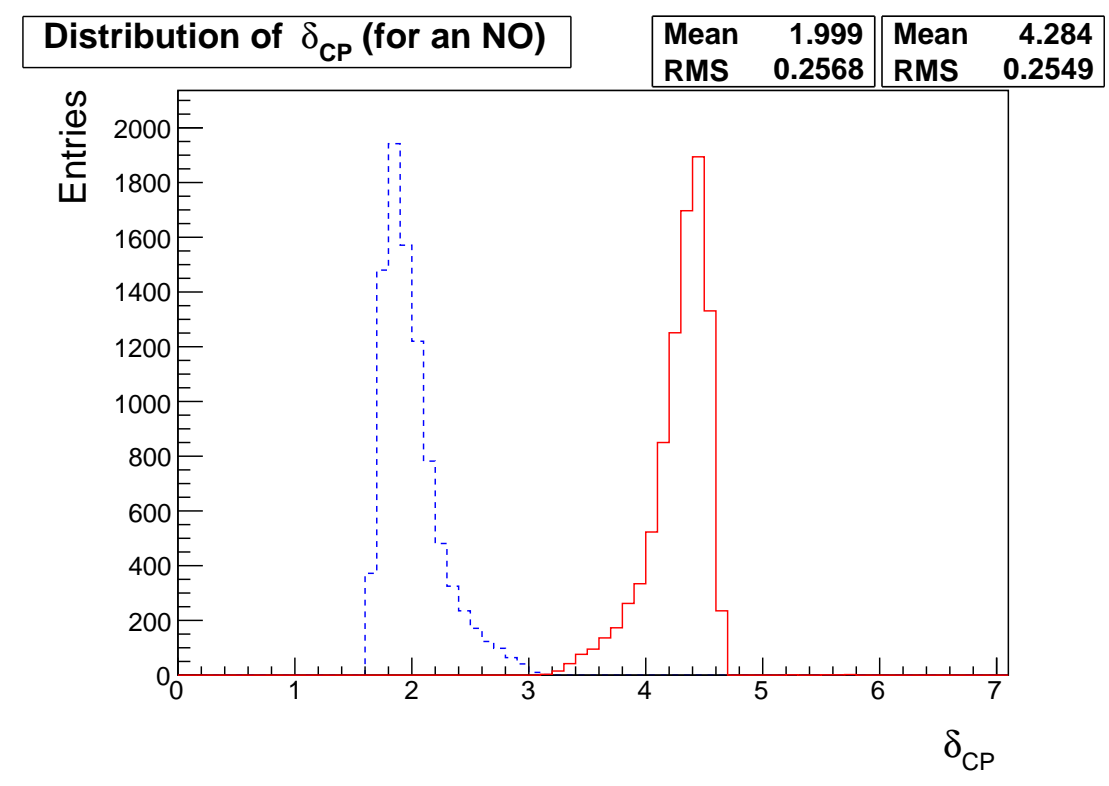

Fig. 1. Distribution of $\delta_{C P}$ for a normal ordering. 


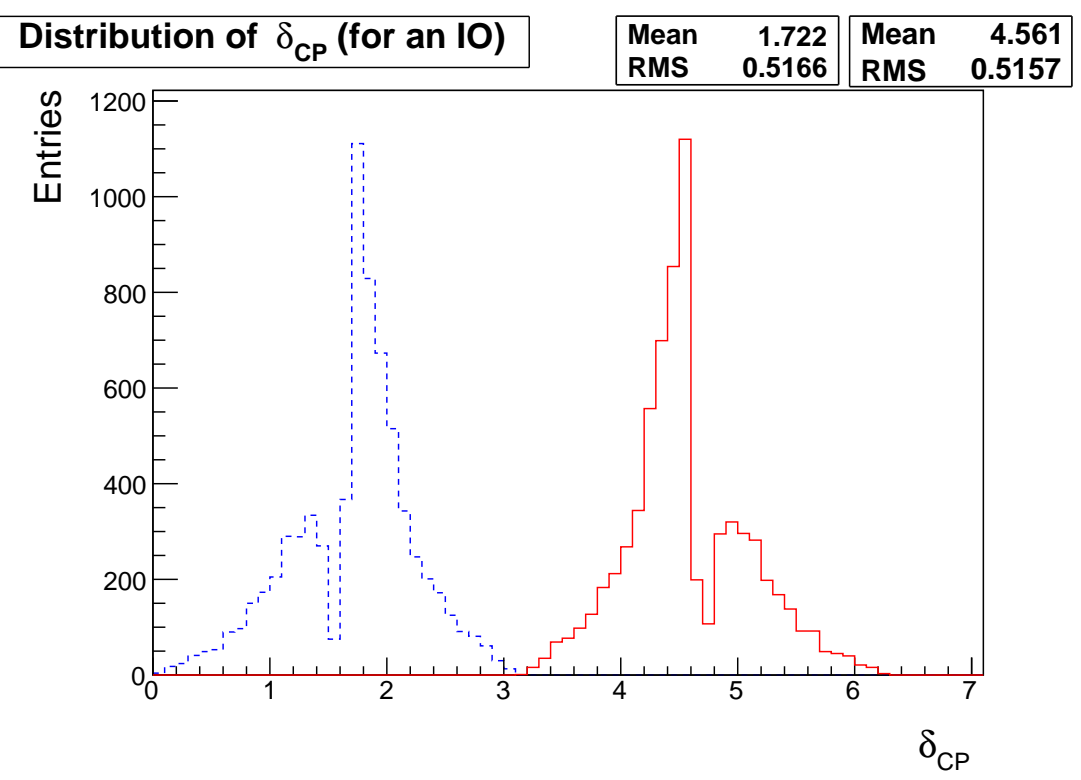

Fig. 2. Distribution of $\delta_{C P}$ for an inverse ordering.

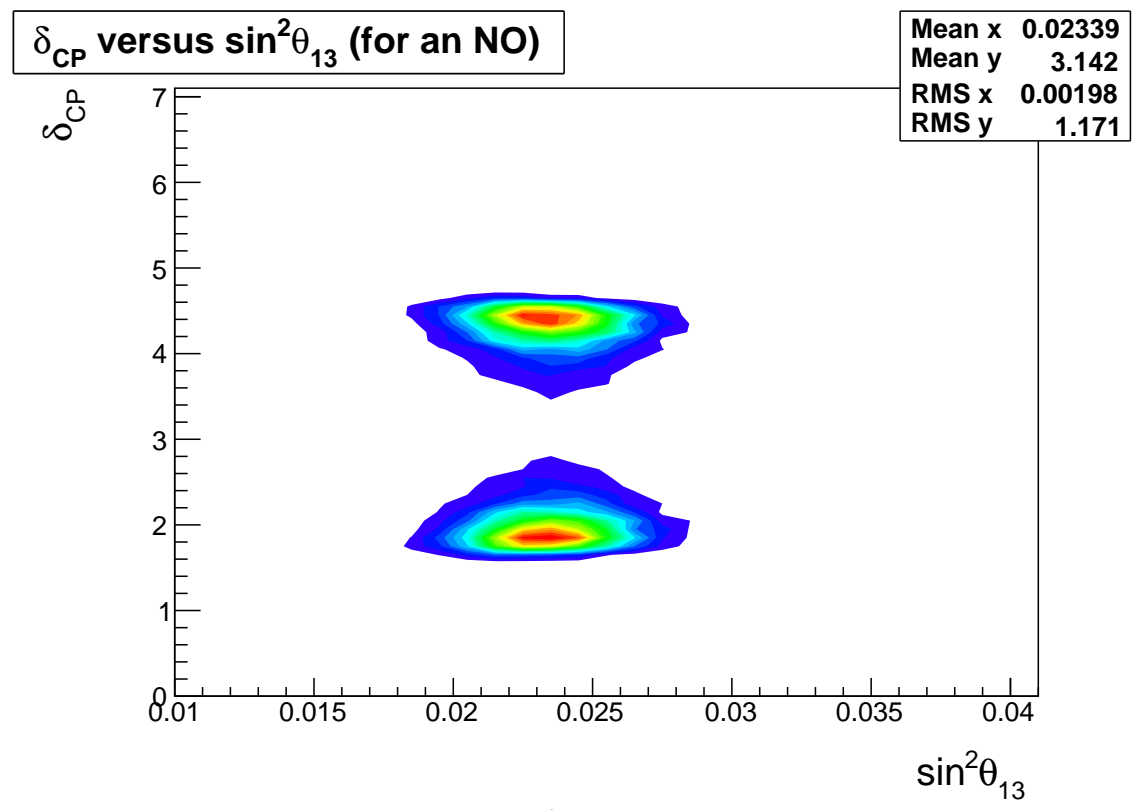

Fig. 3. $\delta_{C P}$ versus $\sin ^{2} \theta_{13}$ for a normal ordering. 


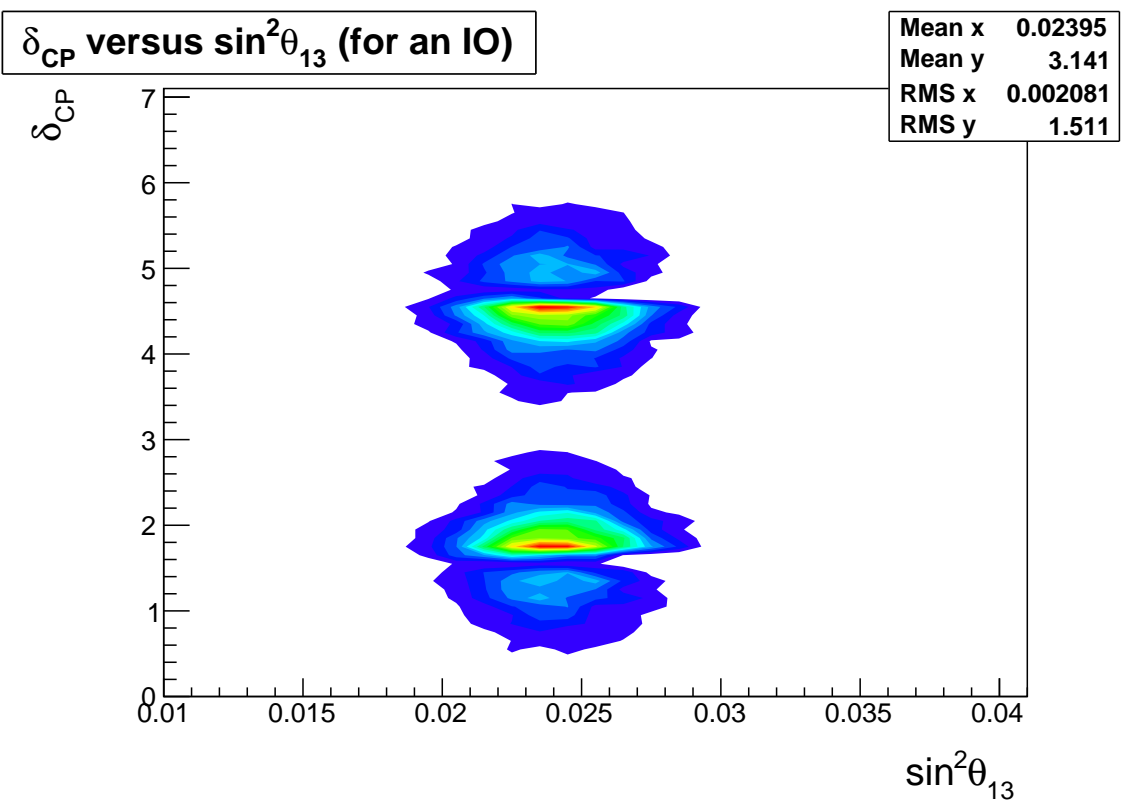

Fig. 4. $\delta_{C P}$ versus $\sin ^{2} \theta_{13}$ for an inverse ordering.

\section{CONCLUSION}

A model of neutrino mass and mixing with an A4 flavor symmetry is considered. The mass matrix is diagonalized and the mixing matrix is found by using a perturbation method. Then, a relation between the CP-violation Dirac phase $\delta_{C P}$ and the mixing angles $\theta_{i j}$ can be established. It allows us to determine $\delta_{C P}$ via the experimental data on $\theta_{i j}$. The result is quite good (near the $1 \sigma$ region of the global fit) for both the normal- and inverse- ordering of the neutrino masses $[12,13]$, therefore, the neutrino mass and mixing matrices are more explicitly fixed.

In this paper a first order perturbation is used but a higher order perturbation is also being considered.

\section{ACKNOWLEDGMENT}

This work is supported by Vietnam's National Foundation for Science and Technology Development (NAFOSTED) under the grant No 103.03-2012.49. The authors would like to thank Dinh Nguyen Dinh and Nguyen Anh Ky for useful discussions and collaboration, and Nguyen Anh Ky for reading the manuscript and comments.

\section{REFERENCES}

[1] H. K. Quang and P. X. Yem, Elementary Particles and Their Interactions: Concepts and Phenomena, SpringerVerlag, Berlin, 1998.

[2] D. J. Griffiths, Introduction to Elementary Particles, John Wiley \& Sons, New York, 1987.

[3] T. P. Cheng and L.F. Li, Gauge theory of elementary particle physics, Oxford university press, Oxford, 2006. 
[4] M. E. Peskin and D. V. Schroeder, An introduction to quantum field theory, Addison-Wesley publishing company, Reading, Massachusetts, 1995.

[5] ATLAS Collaboration, G. Aad et al., Phys. Lett. B 716 (2012) 1, arXiv:1207.7214 [hep-ex].

[6] CMS Collaboration, S. Chatrchyan et al., Phys. Lett. B 716 (2012) 30, arXiv:1207.7235 [hep-ex].

[7] Nguyen Anh Ky and Nguyen Thi Hong Van, Commun. Phys. 25 (2015) 1, arXiv:1503.08630 [hep-ph].

[8] S. Bilenky, Introduction to the physics of massive and mixed neutrinos, Berlin: Springer, 2010.

[9] C. Giunti, C. W. Kim, Fundamentals of neutrino physics and astrophysics, New York: Oxford university Press, 2007.

[10] G. Altarelli, F. Feruglio and L. Merlo, Fortsch. Phys. 61 (2013) 507, arXiv:1205.5133 [hep-ph].

[11] P. M. Ferreira, L. Lavoura and P. O. Ludl, Phys. Lett. B 726 (2013) 767, arXiv:1306.1500 [hep-ph].

[12] K. A. Olive et al., Review of Particle Physics, (Particle Data Group Collaboration) Chin. Phys. C 38 (2014) 090001.

[13] F. Capozzi, G. L. Fogli, E. Lisi, A. Marrone, D. Montanino and A. Palazzo, Phys. Rev. D 89 (2014) 093018, arXiv:1312.2878 [hep-ph].

[14] D. N. Nguyen Dinh, N. Anh Ky, P. Quang Văn and N. T. Hồng Vân, J. Phys. Conf. Ser. 627 (2015) 1, 012003.

[15] G. Altarelli, F. Feruglio, L. Merlo and E. Stamou, JHEP 1208 (2012) 021, arXiv:1205.4670 [hep-ph].

[16] S. F. King and C. Luhn, Rept. Prog. Phys. 76 (2013) 056201, arXiv:1301.1340 [hep-ph].

[17] H. Ishimori and E. Ma, Phys. Rev. D 86 (2012) 045030, arXiv:1205.0075 [hep-ph].

[18] E. Ma, A. Natale and A. Rashed, Int. J. Mod. Phys. A 27 (2012) 1250134, arXiv:1206.1570 [hep-ph].

[19] M. Honda and M. Tanimoto, Prog. Theor. Phys. 119 (2008) 583, arXiv:0801.0181 [hep-ph].

[20] Dinh Nguyen Dinh, Nguyen Anh Ky, Phi Quang Văn and Nguyen Thi Hong Vân, arXiv:1602.07437 [hep-ph].

[21] B. Brahmachari and A. Raychaudhuri, Phys. Rev. D 86 (2012) 051302, arXiv:1204.5619 [hep-ph]. 
